\title{
The Staphylococcus aureus scdA gene: a novel locus that affects cell division and morphogenesis
}

\author{
Eric W. Brunskill, ${ }^{1} \nmid$ Boudewijn L. M. de Jonge ${ }^{2} \ddagger$ and Kenneth W. Bayles ${ }^{3}$
}

Author for correspondence: Kenneth W. Bayles. Tel: +1208885 7966. Fax: +1 2088856518. e-mail: kbayles@uidaho.edu

1 University of Maryland, Baltimore County, Program in Molecular and Cell Biology, Baltimore, MD 21228, USA

2 Laboratory of Microbiology, Rockefeller University, New York, NY 10021, USA

3 Department of Microbiology, Molecular Biology and Biochemistry, University of Idaho, Moscow, ID 83844-3052. USA

\begin{abstract}
A new Staphylococcus aureus gene termed scdA was found upstream of the autolysis regulatory genes, lytS and IytR, and was shown to potentially encode a hydrophilic 25 kDa protein. Analysis of ScdA transcription revealed that it is transcribed as a monocistronic message and is lytSR-independent. A role in cell wall metabolism was indicated by examination of the scdA mutant $S$. aureus KB323, which had a grossly aberrant cellular morphology and formed large cell clusters when grown in liquid culture medium. Furthermore, KB323 exhibited a reduced rate of autolysis and had increased peptidoglycan cross-linking compared to the parental strain, NCTC 8325-4. These data suggest that scdA plays an important role in staphylococcal cell division.
\end{abstract}

Keywords: Staphylococcus aureus, scdA, peptidoglycan cross-linking, septum formation

\section{INTRODUCTION}

Recently, a new two-component regulatory system, comprising LytS and LytR, has been identified in Staphylococcus aureus and shown to be involved in the regulation of autolysis (Brunskill \& Bayles, 1996a). This was first demonstrated by analysis of the $S$. aureus mutant strain KB300, which contained a disruption of the lyt $\mathrm{S}$ gene (Brunskill \& Bayles, 1996a). Autolysis assays revealed that $\mathrm{KB} 300$ lysed at a much faster rate than the parental strain, NCTC 8325-4 (Brunskill \& Bayles, 1996a). These data suggested that LytS and LytR regulate the expression of genes encoding murein hydrolases or genes involved in murein hydrolase activity. This prediction was supported by a zymographic analysis which demonstrated that the lytS mutation caused a dramatic reduction in expression of cytoplasmic and extracellular murein hydrolases, with a corresponding increase in murein hydrolase activity in the cell wall fraction (Brunskill \& Bayles, 1996a). It was hypothesized that the increased rate of autolysis in the

\footnotetext{
†Present address: The Children's Hospital Research Foundation Department of Developmental Biology, 3333 Burnet Ave, Cincinnati, OH 45229-3039, USA.

¥Present address: Astra Research Center Boston, Inc., 128 Sidney St, Cambridge, MA 02139-4239, USA.

The GenBank accession number for the nucleotide sequence determined in this work is U57060.
}

lytS mutant was a result of the overexpression of the cell wall-associated murein hydrolases.

The sequence analysis of the lyt $\mathrm{SR}$ region revealed the presence of two additional open reading frames, designated $\operatorname{lrg} A$ and $\operatorname{lrg} B$, that are located immediately downstream of and are oriented in the same direction as lytS and lytR (Brunskill \& Bayles, 1996b). A Northern blot analysis of RNA isolated from NCTC $8325-4$ and KB300 revealed that $\operatorname{lrg} A$ and $\operatorname{lrg} B$ are cotranscribed and that their transcription is dependent on $l y t S$ and $l y t R$ (Brunskill \& Bayles, 1996b). Studies of $\operatorname{lrg} A$ and $\operatorname{lrg} B$ function suggested that these genes encode a holin-like protein and a gene involved in murein hydrolase activity, respectively (Brunskill \& Bayles, 1996b). Upstream of lytS and lytR was another open reading frame of unknown function. To determine if this gene (designated $s c d A$ ) was also involved in cell wall metabolism, the $s c d A$ open reading frame was insertionally inactivated and the resulting mutant strain was analysed. The results of this analysis, reported here, demonstrate that $s c d A$ affects peptidoglycan cross-linking and is required for normal cell growth and development.

\section{METHODS}

Strains, plasmids and growth conditions. The $S$. aureus strains and plasmids used in this study are listed in Table 1. The Escherichia coli strain used was DH5 $\alpha$. L-broth and Trypticase-soy broth (TSB) were used for cultivation of $E$. coli and $S$. aureus, respectively. Ampicillin $\left(50 \mu \mathrm{g} \mathrm{ml}^{-1}\right)$ and ery- 
Table 1. Strains and plasmids

\begin{tabular}{|lll|}
\hline Strain or plasmid & \multicolumn{1}{c|}{ Description } & \multicolumn{1}{c|}{ Reference } \\
\hline S. aureus & & \\
NCTC $8325-4$ & Wild-type strain cured of prophage & Novick (1990) \\
RN4220 & NCTC 8325-4 $r^{-}$ & Novick et al. $(1990)$ \\
KB320 & RN4220(pORF-2) & This study \\
KB321 & NCTC 8325-4(pORF-2) & This study \\
KB323 & $s c d A::$ pORF-2 integrant & This study \\
KB300 & lytS::pKIN1 integrant & Brunskill \& Bayles \\
& & $(1996 a)$ \\
Plasmids & & \\
pER924 & Ap ${ }^{\mathrm{r} \text { Erm }}$; shuttle/integration vector & Brunskill \& Bayles \\
pORF-2 & pER924 containing a 211 bp internal & (1996a) \\
& $s c d A$ fragment & This study \\
\hline
\end{tabular}

thromycin $\left(2 \mu \mathrm{g} \mathrm{ml}^{-1}\right)$ were used as indicated for selection in $E$. coli and $S$. aureus, respectively.

DNA manipulations. DNA restriction digestions and ligations were performed as described by Sambrook et al. (1989), using enzymes obtained from Promega. The scdA-specific probe (nt 613-823) used in the Northern blot analysis (see below) was PCR-amplified from $S$. aureus genomic DNA using sequence-specific oligonucleotides and Taq polymerase (Promega). PCR products were gel-purified using $\beta$-agarase as described by the manufacturer (New England Biolabs) and radiolabelled with $\left[\gamma-{ }^{32} \mathrm{P}\right]$ ATP (Sambrook et al., 1989). Plasmid transformations of $E$. coli were carried out using the SEM method (Inoue et al., 1990). E. coli plasmid DNA was isolated using the alkaline lysis method followed by $\mathrm{CsCl}$ /ethidium bromide density-gradient centrifugation (Sambrook et al., 1989). S. aureus genomic DNA was isolated as described by Dyer \& Iandolo (1983) and plasmid DNA transformations of $S$. aureus were carried out using a Bio-Rad Gene Pulser (Kraemer \& Iandolo, 1990). Transduction in S. aureus strains was performed using $\phi 11$ as described by Shafer $\&$ Iandolo (1979).

Campbell integration. The $s c d A$ gene disruption was created using a Campbell-like integration mechanism by cloning the $211 \mathrm{nt} s c d A$ PCR product (see above) into the shuttle vector pER924 (Brunskill \& Bayles, 1996a). The resulting plasmid, pORF-2, was electroporated into $S$. aureus RN4220 at the permissive temperature $\left(30^{\circ} \mathrm{C}\right)$ to generate strain $\mathrm{KB} 320$. The plasmid was then transferred into NCTC $8325-4$ by transduction using the bacteriophage $\phi 11$, generating $S$. aureus strain KB321. An overnight culture of KB321, grown at the permissive temperature $\left(30^{\circ} \mathrm{C}\right)$, was streaked on Trypticasesoy agar containing erythromycin and incubated overnight at the nonpermissive temperature $\left(43^{\circ} \mathrm{C}\right)$. Since plasmid replication is inhibited at $43^{\circ} \mathrm{C}$, only those cells that had undergone a Campbell-like integration event into the $s c d A$ gene were selected. After overnight growth, single colonies were restreaked and grown again at $43^{\circ} \mathrm{C}$. Chromosomal DNA from single colonies was isolated and a Southern analysis was used to confirm that the clones contained a $s c d A$ gene disruption (data not shown). One such clone was identified and designated KB323.

DNA sequencing and computer analysis. The nucleotide sequence of $s c d A$ was determined using the dideoxy chaintermination method (Sanger et al., 1977) and the Sequenase DNA sequencing kit (United States Biochemical). Sequence- specific oligonucleotide primers were synthesized using an Expedite 8909 nucleotide synthesizer and were utilized to sequence both strands. A computer analysis of the sequences generated was conducted using the GCG Wisconsin Computer Package Unix Version 8.0 (Devereux et al., 1984).

RNA analysis. S. aureus RNA $(20 \mu \mathrm{g})$ was isolated from exponentially growing and stationary-phase cells as described by Hart et al. (1993) and was analysed by Northern blotting (Sambrook et al., 1989) using a scdA-specific probe. A primer extension analysis of the $s c d A$ promoter region was conducted using the procedure described previously (Brunskill \& Bayles, 1996a) and an oligonucleotide primer that was complementary to the $5^{\prime}$ end of the $s c d A$ gene (nt 407-437).

Transmission electron microscopy. Mid-exponential-phase NCTC 8325-4 and KB323 cells were resuspended in $2.5 \%$ glutaraldehyde in Dulbecco's phosphate-buffered saline (PBS) overnight. The cells were collected and resuspended in five drops of PBS. The cells were then vortexed and incubated for $5 \mathrm{~min}$ at room temperature. Next, the cells were pelleted, resuspended in $1.5 \mathrm{ml} \mathrm{PBS}$ containing $1 \% \mathrm{OsO}_{4}$, and incubated for $30 \mathrm{~min}$ at room temperature. This was followed by centrifugation for $5 \mathrm{~min}$ at $13500 \mathrm{~g}$. The cell pellet was washed with $1.5 \mathrm{ml}$ PBS, dehydrated in a series of ethanol washes and then fixed in propylene oxide/Araldite $502(1: 1, \mathrm{v} / \mathrm{v})$ for $30 \mathrm{~min}$, followed by a treatment with propylene oxide/ Araldite $502(1: 3, \mathrm{v} / \mathrm{v})$ for $60 \mathrm{~min}$. The pellets were then embedded in polyethylene embedding capsules and spun in a clinical centrifuge on an embedding trench for $3 \mathrm{~min}$. The pellets were incubated at $60^{\circ} \mathrm{C}$ for $18 \mathrm{~h}$ and cut on a Sorvall MT2B ultramicrotome at a thickness of $60 \mathrm{~nm}$. The thin sections were stained with $1 \%$ uranyl acetate/lead acetate and viewed with a Zeiss 10-CA transmission electron microscope.

Autolysis assays. Autolysis assays were performed as described by Mani et al. (1993). Cell samples $(50 \mathrm{ml})$ were collected from exponentially growing TSB cultures $\left(\mathrm{OD}_{580}\right.$ $0.7)$ containing $1 \mathrm{M} \mathrm{NaCl}$ and the cells were pelleted by centrifugation. The cells were washed twice with $50 \mathrm{ml}$ icecold water and resuspended in $50 \mathrm{ml} 0.05 \mathrm{M} \mathrm{Tris} / \mathrm{HCl}$ ( $\mathrm{pH} 7.2$ ) containing $0.05 \%$ Triton $\mathrm{X}-100$ (Sigma). The cells were then incubated at $30^{\circ} \mathrm{C}$ with shaking, and the $\mathrm{OD}_{580}$ was measured at $30 \mathrm{~min}$ intervals.

Peptidoglycan analysis. S. aureus peptidoglycan was prepared, digested into muropeptides and analysed using reversephase HPLC as described by de Jonge et al. (1992). 


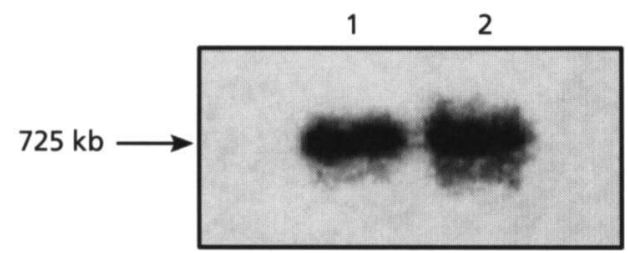

Fig. 1. Northern blot analysis. Total cellular RNA from strains NCTC 8325-4 (lane 1) and KB300 (lane 2) was separated in a formaldehyde-agarose gel, transferred to nitrocellulose paper and hybridized with a scdA-specific probe. Transcript size was determined by comparison with RNA size markers.

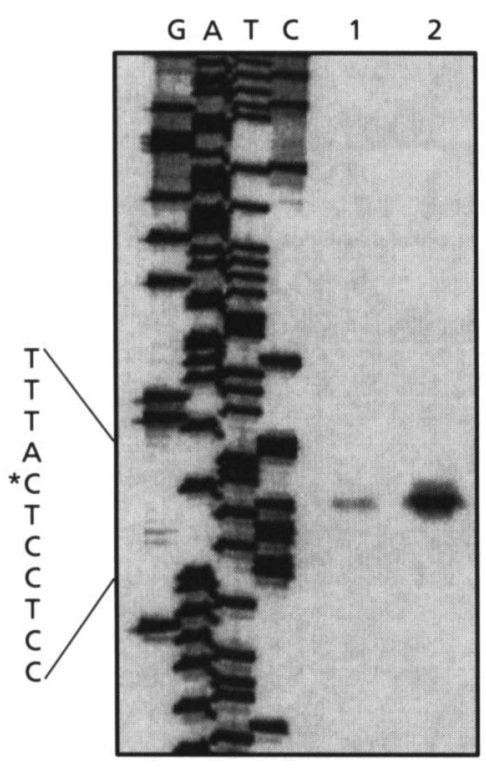

Fig. 2. Transcription start site of scdA. Total cellular RNA $(25 \mu \mathrm{g})$ was isolated (Hart et al., 1993) from exponentially growing (lane 1) and stationary-phase (lane 2) cells of $S$. aureus NCTC 8325-4 and subjected to primer extension analysis (Brunskill \& Bayles, 1996a). The sizes of the extended products were determined by comparison with a DNA sequencing ladder of the $\operatorname{scd} A$ promoter region that was generated using the same primer.

\section{RESULTS AND DISCUSSION}

\section{Sequence analysis of the scdA gene}

Analysis of the DNA sequence upstream of the $S$. aureus autolysis regulatory genes, $l y t S$ and $l y t R$ (Brunskill $\&$ Bayles, 1996a), revealed the presence of a previously uncharacterized open reading frame which we termed $s c d A$. The $s c d A$ coding sequence is preceded by a consensus ribosome-binding site and potentially encodes a hydrophilic protein ( $\mathrm{ScdA}$ ) containing 224 amino acids and having a deduced molecular mass of $25369 \mathrm{Da}$. Immediately downstream from $s c d A$ is a large $12 \mathrm{bp}$ inverted repeat sequence that is a potential rho-independent terminator. Upstream of $s c d A$ is another open reading frame that is also followed by a potential rho-independent terminator. The ScdA protein shares

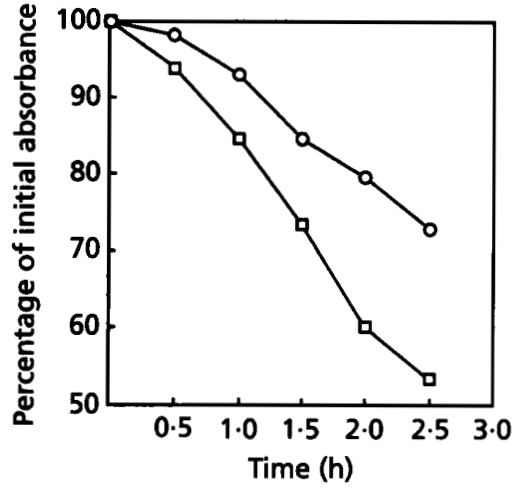

Fig. 3. Autolysis assay of S. aureus NCTC 8325-4 ( $\square$ ) and KB323 (O). The rate of autolysis was measured as the decline in $O D_{580}$ versus time. The data presented illustrate one of three independent experiments which exhibited the same difference betwen the autolysis rates of the two strains.

the greatest amino acid sequence similarity $(26.9 \%$ and $27.2 \%$ identical residues, respectively) with the hypothetical E. coli and Haemophilus influenzae YtfE proteins (accession nos P39313 and P45312, respectively), whose functions have not been determined. The deduced amino acid sequence of the upstream open reading frame did not match any sequences in the GenBank database.

\section{The transcription of $\operatorname{scd} A$}

To determine if $s c d A$ transcription is affected by lytS and $l y t R$, a Northern blot analysis of RNA isolated from $S$. aureus NCTC 8325-4 and its $l y t S$ mutant derivative, $\mathrm{KB} 300$, was performed using a $s c d A$-specific probe. This analysis revealed the presence of an approximately $0.7 \mathrm{~kb} \mathrm{scdA}$-specific transcript that was present in both NCTC 8325-4 and KB300 (Fig. 1, lanes 1 and 2, respectively), indicating that $s c d A$ transcription is independent of $l y t S$ and $l y t R$. Furthermore, a primer extension analysis localized the $s c d A$ transcription start site to a guanine residue 16 nucleotides $5^{\prime}$ relative to the putative $s c d A$ translation start codon (Fig. 2). Upstream of the transcription start site is a putative $\sigma^{\mathrm{A}}$ promoter region (TTCATT- $\mathrm{N}_{18}$-TATACA), which matches 7 out of 12 bases of the prototypical $\sigma^{\mathrm{A}}$ promoter of Bacillus subtilis (Moran, 1993). These data, along with the location of the potential rho-independent terminator sequence, are consistent with the size of the $s c d A$ transcript and suggest that $s c d A$ is transcribed as a monocistronic message. This analysis also revealed that a much stronger signal was produced using RNA from stationary-phase cells (Fig. 2, lane 2) compared to midexponential-phase cells (Fig. 2, lane 1). As equal amounts of RNA were used in the primer extension experiments, these data indicate that significantly more $s c d A$ message is present in stationary-phase cells than in mid-exponential-phase cells and that this gene is subject to temporal regulation. 

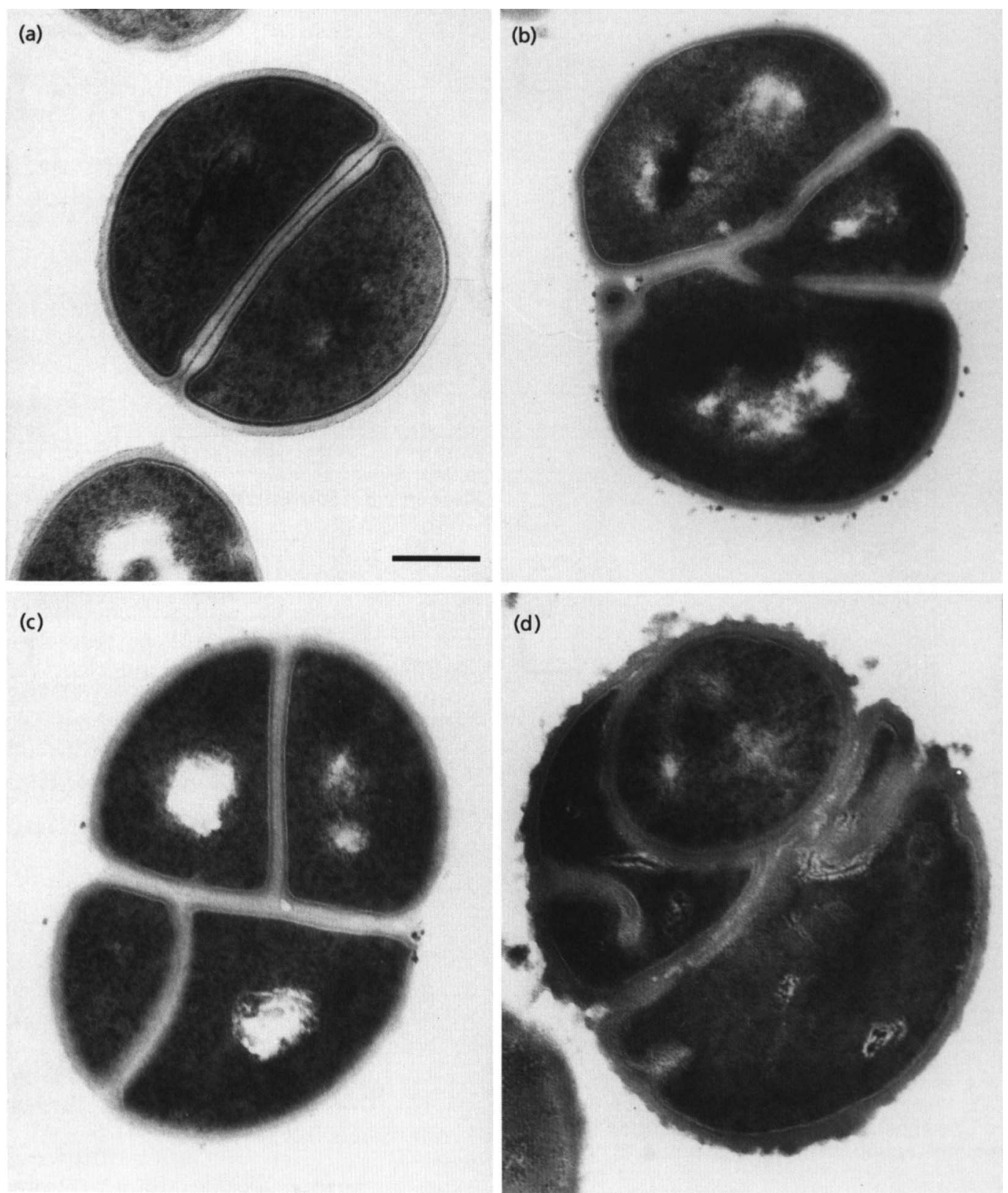

Fig. 4. Transmission electron micrographs of cells of $S$. aureus NCTC $8325-4$ (a) and KB323 (b-d). Bar, $0.2 \mu \mathrm{m}$ (magnification is the same in each panel).

\section{Analysis of a sedA mutation}

To investigate the function of $s c d A$, an internal fragment from this gene was used to construct an insertional $s c d A$ disruption in NCTC 8325-4, creating strain KB323 (see Methods). One striking feature of KB323 was that it formed large aggregated clumps of cells when grown in liquid culture medium, a feature that is characteristic of $S$. aureus mutants deficient in murein hydrolase production. For example, Oshida et al. (1995) have recently characterized the atl gene, which encodes a bifunctional, extracellular protein that is proteolytically processed to generate an endo- $\beta$ - $N$-acetylglucosaminidase and an $N$ -
acetylmuramyl-L-alanine amidase. A mutation within atl results in cells that form large clusters (Oshida et al., 1995; Oshida \& Tomasz, 1992) and exhibit a reduced rate of autolysis (Foster, 1992). It was hypothesized that these two murein hydrolases are required for the separation of cells by cleaving the peptidoglycan connecting the two adjoining daughter cells (Sugai et al., $1989,1995)$. An autolysis assay of KB323 demonstrated that this strain also undergoes autolysis at a lower rate than the parental strain (Fig. 3), indicating that $s c d A$ could affect murein hydrolase activity or that the mutation has a polar effect on the downstream autolysis regulatory genes (Brunskill \& Bayles, 1996a). However, 


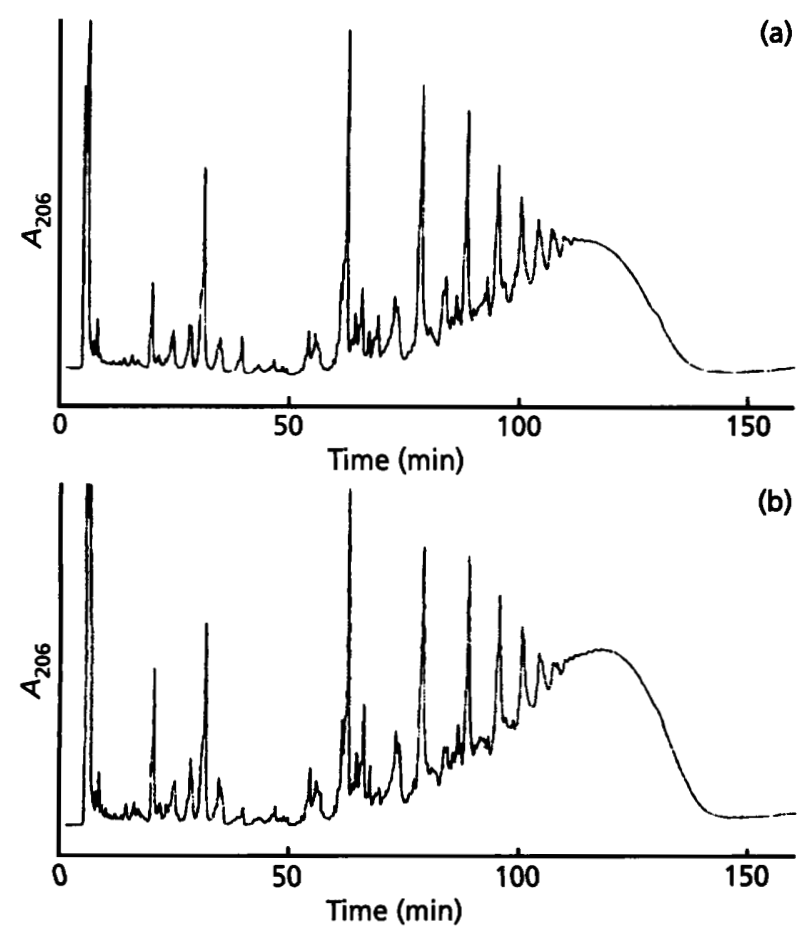

Fig. 5. Muropeptide profiles of S. aureus strains. Peptidoglycan was obtained from S. aureus strains NCTC 8325-4 (a) and KB323 (b) and digested with muramidase; the resulting muropeptides were separated by reverse-phase HPLC.

this reduced autolysis rate did not appear to be the result of altered murein hydrolase activity since no difference in the zymographic profiles of NCTC 8325-4 and KB323 could be detected (data not shown). Therefore, it is possible that the decreased autolysis rate exhibited by $\mathrm{KB} 323$ arose as a result of structural changes within the peptidoglycan.

To examine KB323 cells more closely, transmission electron microscopy was utilized; it revealed that the scdA mutation brought about dramatic defects in cellular morphology (Fig. 4). These defects appeared to be caused by the aberrant placement of septa resulting in misplaced, multiple and/or aborted cross-wall formation. In addition, the cell wall of KB323 was rough and diffuse compared to the parental strain. Interestingly, the aberrant cellular morphology of KB323 was strikingly similar to $S$. aureus cells carrying $\mathrm{fem} A$ or $\mathrm{femB}$ mutations (Henze et al., 1993). These mutants also exhibited reduced autolysis, which is probably due to the reduced glycine content of the their pentapeptide cross-bridges (de Jonge et al., 1993; Henze et al., 1993; Maidhof et al., 1991).

\section{Cell wall analysis}

To determine the composition of NCTC $8325-4$ and KB323 peptidoglycan, cell walls were prepared and hydrolysed, and the resulting muropeptides were analysed using reverse-phase HPLC as described by de
Jonge et al. (1992). Although the composition of the cross-bridges appeared to be unaffected by the $s c d A$ mutation, the total amount of peptidoglycan crosslinking in KB323 was higher than in NCTC 8325-4 (Fig. 5). In KB323 cells, $44 \%$ of the muropeptides (expressed as a percentage of total UV absorption) were found in highly cross-linked form ( $>$ octamers), compared to $37 \%$ in strain NCTC 8325-4. Whether this increase in cross-linking is responsible for the decreased autolysis rate of this mutant is not known. Since fem $A$ and femB mutant cells have similar morphological abnormalities to $\mathrm{KB} 323$, but their peptidoglycan exhibited decreased cross-linkage and a reduced glycine content (de Jonge $e t$ al., 1993; Henze et al., 1993; Maidhof et al., 1991), these morphological abnormalities may be the result of a factor(s) unrelated to the physical state of the cell wall.

Although the precise function of $s c d A$ is unknown, the data generated by this study demonstrate that it can be added to the growing list of cell-wall-related genes in this gene cluster. In spite of the fact that the transcription of $s c d A$ was unaffected by LytS and LytR, scdA was clearly required for normal peptidoglycan metabolism and cell development, indicating that this region of the chromosome is involved in different aspects of cell wall physiology. Continued analysis of these, and additional genes in this region are ongoing in our laboratory and will undoubtably lead to further insight into the regulation of the components necessary for peptidoglycan assembly and processing in $S$. aureus.

\section{ACKNOWLEDGEMENTS}

We thank Ron Yasbin and Hal Schreier for critical reading and insightful comments on this manuscript. This work was supported by USDA grant no. 9304112.

\section{REFERENCES}

Brunskill, E. W. \& Bayles, K. W. (1996a). Identification and molecular characterization of a putative regulatory locus that affects autolysis in Staphylococcus aureus. J Bacteriol 178, 611-618.

Brunskill, E. W. \& Bayles, K. W. (1996b). Identification of LytSRregulated genes from Staphylococcus aureus. J Bacteriol 178, $5810-5812$.

Devereux, J., Haeberli, P. \& Smithies, O. (1984). A comprehensive set of sequence analysis progams for the VAX. Nucleic Acids Res $12,387-395$.

Dyer, D. W. \& landolo, J. J. (1983). Rapid isolation of DNA from Staphylococcus aureus. Appl Environ Microbiol 46, 283-285.

Foster, S. J. (1992). Analysis of the autolysins of Bacillus subtilis 168 during vegetative growth and differentiation by using renaturing polyacrylamide gel electrophoresis. J Bacteriol 174, $464-470$.

Hart, M. E., Smeltzer, M. S. \& landolo, J. J. (1993). The extracellular protein regulator $(x p r)$ affects exoprotein and $a g r$ mRNA levels in Staphylococcus aureus. J Bacteriol 175, 7875-7879.

Henze, U., Sidow, T., Wecke, J., Labischinski, H. \& Berger-Bachi, B. (1993). Influence of $f e m B$ on methicillin resistance and peptidoglycan metabolism in Staphylococcus aureus. J Bacteriol 175, 1612-1620. 
Inoue, H., Nojima, H. \& Okayama, H. (1990). High efficiency transformation of Escherichia coli with plasmids. Gene 96, 23-28.

de Jonge, B. L., Chang, Y. S., Gage, D. \& Tomasz, A. (1992). Peptidoglycan composition of a highly methicillin-resistant Stapbylococcus aureus strain. The role of penicillin binding protein 2A. J Biol Chem 267, 11248-11254.

de Jonge, B. L., Sidow, T., Chang, Y. S., Labischinski, H., BergerBachi, B., Gage, D. A. \& Tomasz, A. (1993). Altered muropeptide composition in Staphylococcus aureus strains with an inactivated femA locus. J Bacteriol 175, 2779-2782.

Kraemer, G. R. \& landolo, J. J. (1990). High-frequency transformation of Staphylococcus aureus by electroporation. Curr Microbiol 21, 373-376.

Maidhof, H., Reinicke, B., Blumel, P., Berger-Bachi, B. \& Labischinski, H. (1991). femA, which encodes a factor essential for expression of methicillin resistance, affects glycine content of peptidoglycan in methicillin-resistant and methicillin-susceptible Staphylococcus aureus strains. J Bacteriol 173, 3507-3513.

Mani, N., Tobin, P. \& Jayaswal, R. K. (1993). Isolation and characterization of autolysis-defective mutants of Staphylococcus aureus created by Tn917-lacZ mutagenesis. J Bacteriol 175, 1493-1499.

Moran, C. P., Jr (1993). RNA polymerase and transcription factors. In Bacillus subtilis and Other Gram-positive Bacteria, pp. 653-667. Edited by A. L. Sonenshein, J. A. Hoch \& R. Losick. Washington, DC: American Society for Microbiology.

Novick, R. P. (1990). The staphylococcus as a molecular genetic system. In Molecular Biology of the Staphylococci, pp. 1-37. Edited by R. P. Novick. New York: VCH Publishers.

Novick, R., Kornblum, J., Kreisworth, B., Projan, S. \& Ross, H. (1990). Regulation of post-exponential phase exoprotein synthesis in Staphylococcus aureus. In Microbial Determinants of Viru- lence and Host Response, pp. 3-18. Edited by E. M. Ayoub, G. H. Cassell, W. C. Branche \& T. J. Henry. Washington, DC: American Society for Microbiology.

Oshida, T. \& Tomasz, A. (1992). Isolation and characterization of a Tn551-autolysis mutant of Staphylococcus aureus. J Bacteriol 174, 4952-4959.

Oshida, T., Sugai, M., Komatsuzawa, H., Hong, Y. M., Suginaka, H. \& Tomasz, A. (1995). A Staphylococcus aureus autolysin that has an $\mathrm{N}$-acetylmuramoyl-L-alanine amidase domain and an endo-beta- $N$-acetylglucosaminidase domain: cloning, sequence analysis, and characterization. Proc Natl Acad Sci USA 92, 285-289.

Sambrook, J., Fritsch, E. F. \& Maniatis, T. (1989). Molecular Cloning : a Laboratory Manual. Cold Spring Harbor, New York: Cold Spring Harbor Laboratory.

Sanger, F., Nicklen, S. \& Coulson, A. R. (1977). DNA sequencing with chain-terminating inhibitors. Proc Natl Acad Sci USA 74, 5463-5467.

Shafer, M. W. \& landolo, J. J. (1979). Genetics of staphylococcus enterotoxin B in methicillin-resistant isolates of Staphylococcus aureus. Infect Immun 25, 902-911.

Sugai, M., Koike, H. H., Miyake, Y., Nogami, R. \& Suginaka, H. (1989). Purification of a $51 \mathrm{kDa}$ endo-beta-acetylglucosaminidase from Staphbylococcus aureus. FEMS Microbiol Lett 61, 267-272.

Sugai, M., Komatsuzawa, H., Akiyama, T., Hong, Y., Oshida, T., Miyake, Y., Yamaguchi, T. \& Suginaka, H. (1995). Identification of endo-beta- $N$-acetylglucosaminidase and $N$-acetylmuramyl-Lamidase as cluster-dispersing enzymes in Stapbylococcus aureus. J Bacteriol 177, 1491-1496.

Received 26 February 1997; revised 7 May 1997; accepted 6 June 1997. 